\title{
Clomiphene-induced pituitary apoplexy in a patient with acromegaly
}

\author{
AB Walker, PR Eldridge, IA MacFarlane
}

\begin{abstract}
Summary
We describe the case of an acromegalic woman with primary infertility who was treated with clomiphene, and subsequently developed pituitary apoplexy.
\end{abstract}

Keywords: acromegaly, pituitary apoplexy, clomiphene

The causes and clinical features of pituitary apoplexy are described in boxes 1 and 2 . We describe a case in an acromegalic woman who was receiving clomiphene.

\section{Case report}

A 24-year-old woman presented with primary infertility. Her periods were regular every 28 days, lasting five days and menarche had occurred aged 13. She had not used oral contraceptives. Her basal serum hormone levels were: prolactin $599 \mathrm{mU} / 1$, luteinising hormone (LH) 4.7 IU/1, follicle-stimulating hormone (FSH) 3.6 IU/1, thyroid-stimulating hormone (TSH) $0.5 \mathrm{mU} / 1$, free thyroxine $16 \mathrm{pmol} / 1$, tes-

\begin{tabular}{|l|}
\hline Pituitary apoplexy: causes \\
\hline - haemorrhagic infarction of pituitary tumour \\
- following obstetric haemorrhage \\
anticoagulant therapy \\
other predisposing factors: bleeding \\
pituitary, raised intracranial pressure, \\
carotid angiography, bromocriptine therapy, \\
ventilation, diabetes mellitus mechanical \\
\hline
\end{tabular}

Box 1

Walton Hospital, Rice Lane, Liverpool L9 1AE, UK Department of Endocrinology AB Walker IA MacFarlane Walton Centre for Neurology and Neurosurgery PR Eldridge tosterone $1.1 \mathrm{nmol} / 1$, and progesterone (day 21 ) $2.1 \mathrm{pmol} / 1$. She was prescribed clomiphene $50 \mathrm{mg} /$ day for five days.

Three days after her final tablet of clomiphene, she developed acute severe generalised headache and progressive visual impairment. Visual acuity was reduced (6/18: left eye, 6/36: right eye) with a bitemporal hemianopia and early papilloedema. Skull Xray showed an enlarged pituitary fossa and cranial computed tomography (CT) demonstrated an enhancing mass with considerable supra-sellar extension (figure).

In view of the rapid and progressive visual failure, a neurosurgical opinion was obtained and an emergency transsphenoidal decompression was performed. Necrotic and haemorrhagic debris of pituitary tumour was removed, with immediate improvement in vision postoperatively.

Endocrine assessment postoperatively revealed the clinical features of acromegaly. The diagnosis of active acromegaly was confirmed postoperatively, with elevated random growth hormone levels of $>20 \mathrm{mU} / 1$ (reference range $<10$ ) and an IGF-1 level of $190 \mathrm{nmol} / 1(14-48)$. Serum $\alpha$-subunit was not elevated $(<0.3 \mathrm{IU} / 1)$.

Subsequently, she underwent repeat transsphenoidal removal of residual pituitary tumour followed by external irradiation. Immunohistochemistry of pituitary tissue from the second operation was positive for growth hormone, but negative for FSH, LH, prolactin, TSH and adrenocorticotropin.

\section{Discussion}

Pituitary apoplexy, occurring in patients with pituitary tumours, has been reported following injection of thyrotropin-releasing hormone and after combined pituitary function testing: hypoglycaemia, gonadotropin-releasing hormone (GnRH) and thyrotropin-releasing hormone. ${ }^{1-4}$ In recent reports, pituitary apoplexy occurred following $\mathrm{GnRH}$ injection alone. One was a patient with a probable macroprolactinoma ${ }^{5}$ and the other two were patients with gonadotrophinomas. ${ }^{6,7}$ Pituitary apoplexy in a patient with a previously undiagnosed pituitary tumour occurred nine days after subcutaneous administration of the LHRH analogue, goserelin (Zoladex) ${ }^{8}$ Pituitary apoplexy occurred when peak goserelin levels would have been reached, suggesting that this may have been involved in the mechanism. 
Figure Non-contrast CT scan, demonstrating a midline, hyperdense mass extending towards an expanded sella, consistent with recent haemorrhage into a pituitary adenoma

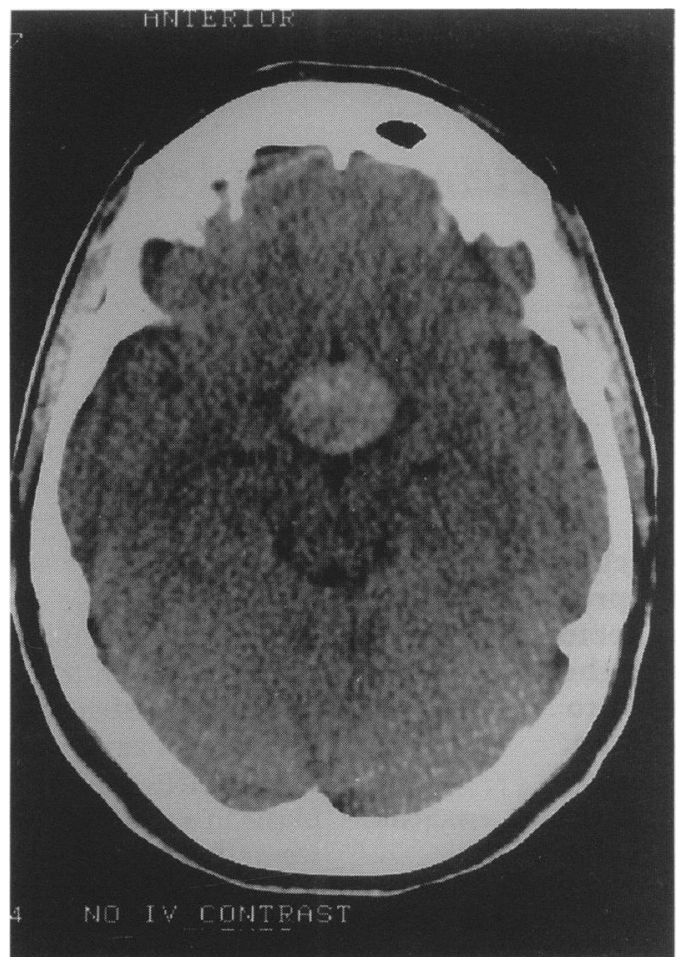

\section{Properties of clomiphene}

- anti-oestrogen, having a similar structure to oestradiol

- acts on the hypothalamus to increase the GnRH pulse frequency resulting in increased $\mathrm{LH}$ (and FSH)

- used in the treatment of anovulation

- multiple pregnancy rate is $8 \%$

- ovarian enlargement, vasomotor flushes and abdominal-pelvic bloating are the commonest adverse effects

- ovarian hyperstimulation occurs rarely

- blurred vision and scintillating scotomata are reported

Box 4

\section{Learning/summary points \\ - pituitary apoplexy is a potentially fatal condition \\ - it may occur following clomiphene therapy in patients with pituitary adenomas \\ - deteriorating visual acuity is an indication for urgent neurosurgical decompression}

\section{Box 5}

\section{Pituitary apoplexy: endocrine} dysfunction

- variable degrees of hypopituitarism

- growth hormone deficiency

- gonadotropin deficiency

- secondary adrenocortical deficiency

- secondary hypothyroidism

- diabetes insipidus (uncommon)

Box 3

Clomiphene citrate is a long-acting antioestrogen and is used in the treatment of anovulation associated with polycystic ovarian disease and in women with normogonadotropic amenorrhoea. Its main mode of action is to bind to oestrogen receptors in the hypothalamus and thus increase the pulse frequency of $\mathrm{GnRH} .{ }^{9}$ This results in a rise in plasma levels of $\mathrm{LH}$ and FSH, which cause follicular development and thereby a rise in serum oestrogen levels and ovulation.

In this patient, clomiphene may have caused infarction of the pituitary tumour by increasing portal blood $\mathrm{GnRH}$ concentrations indirectly,

1 Cimino A, Corsini R, Radaeli E, Bollati A, Giustina G. Transient amaurosis in a patient with pituitary macroadenoma after intravenous gonadotropin and thyrotropin releasing hormones. Lancet 1981; ii: 95.

2 Drury PL, Belchetz PE, McDonald WI, Thomas DGT Besser GM. Transient amaurosis and headache after thyrotropin releasing hormone. Lancet 1982; i: 218-9.

3 Bernstein M, Hegele RA, Gentili F, et al. Pituitary apoplex associated with a triple bolus test. $\mathcal{F}$ Neurosurg 1984; 61: 586-90.

4 Chapman AJ, Williams G, Hockley AD, London DR. Pituitary apoplexy after combined tests of anterior pituitary function. $B M F$ 1985; 291: 26.

5 Arafah BM, Taylor HC, Salazar R, Saadi H, Selman WR Apoplexy of a pituitary adenoma after dynamic testing with gonadotropin-releasing hormone. Am $f \mathrm{Med} 1989 ; 87$ gonado. rather than the immediate increase which would be expected after intravenous injection of GnRH..$^{5-7}$ Peak plasma GnRH levels are achieved approximately 8-10 days after starting clomiphene, when given at a dose of $50 \mathrm{mg}$ daily for five days. ${ }^{10}$ The mechanism by which GnRH may cause pituitary tumour infarction is unclear but may be due to vascular spasm, portal blood being preferentially diverted to pituitary gonadotrophs under the influence of high concentrations of $\mathrm{GnRH}$, with ischaemia and infarction of the somatotrophinoma. Rapid tumour expansion may have been induced by GnRH, resulting in ischaemic necrosis, although there was no evidence of increased mitosis on histological examination. Alternatively, GnRH may have acutely stimulated gonadotrophin synthesis and release, the increase in metabolic activity may have resulted in cellular hypoxia and subsequent infarction.

As far as we are aware, this is the first reported case of clomiphene-induced pituitary apoplexy. Headache and visual symptoms developing within days of clomiphene therapy in patients with pituitary tumours should raise the suspicion of pituitary apoplexy.

6 Masson EA, Atkin SL, Diver M, White MC. Pituitary apoplexy and sudden blindness following the administration apoplexy and sudden blindness following the administration of gonadotropin

7 Korsic M, Lelas-Bahun N, Surdonja P, Besenski N, Horvat $S$, Plasvic V. Infarction of FSH-secreting pituitary adenoma. Acta Endocrinol 1984; 107: 149-54.

8 Ando S, Hoshino T, Mihara S. Pituitary apoplexy after goserelin. Lancet 1995; 345: 458.

9 Kerin JF, Liu JH, Phillipou G, Yens SSC. Evidence for a hypothalamic site of action of clomiphene citrate in women f Clin Endocrinol Metab 1985; 61: 265-8.

10 Miyake A, Aono T, Minagawa J, Kawamura Y, Kurachi K. Changes in plasma luteinizing hormone-releasing hormone during clomiphene-induced ovulatory cycles. Fertil Steril 1980; 34: 172-4. 